CB5-01:

Where Science, Management and Administration Meet: Lessons Learned from Large Multi-site Programs

Kimberly Lane'; Sherry Lee Lauf²; Sarah Madrid³ Leah Tuzzio²

${ }^{1}$ Harvard Pilgrim Health Care Institute, Harvard Medical School;, ${ }^{2}$ Group Health Cooperative; ${ }^{3}$ Kaiser Permanente Colorado

Background/Aims: Within the HMORN multi-site collaborations are routine but as they grow in size and scope, the common challenges of managing these projects becomes more complex. As funders look to the HMORN as a model for distributed research, it is important to document best practices across projects and institutions. Methods: Using examples from across the HMORN, we will explore best practices and common challenges for successfully implementing large scale projects. Topics will include:

- strategies for large scale communication

- working with external partners

- privacy and human subjects

- contracting and budgeting

- general management of large scale network projects.

Each topic will feature examples of common issues across HMORN programs and highlight a lesson learned from the Cancer Research Network, Mini-Sentinel and other large collaborations. Effective innovations or approaches, as well as barriers and challenges will be highlighted. Discussion: As one of the most successful health care research networks, the experience and expertise of the HMORN can provide strategies and guidance to enhance and expand new and ongoing collaborations.

Keywords: Multi-site Collaborations; Program Management; HMORN Administrative and Technical

doi:10.3121/cmr.2012.1100.cb5-01

CB5-02:

New Rules: Dealing With Conflicts of Interest in Public Health Service Funded Research: What Every Institution and Every Investigator Needs to Know

Jeffrey Braff ${ }^{1}$

${ }^{1}$ Kaiser Permanente - Program Offices

Background/Aims: New regulations regarding researcher Conflicts of Interest (COI) for federally funded research have just been promulgated by the Federal Government. All investigators and institutions conducting such research are required to comply with these new COI rules by August 24, 2012. The aims of this presentation are to 1) familiarize investigators and research administrators with these new regulations, and 2) discuss what steps institutions need to take in order to be in compliance. In addition, specific rules dealing with institutional COI that exist nowhere else also are in place for the FDA Mini-Sentinel Project. These rules will be discussed in detail. Methods: This presentation will provide investigators and institutions with a detailed overview of the brave new world of COI and related federal requirements through a combination of didactic learning and interactive Q\&A. Discussion: New COI rules potentially represent significant reporting changes on the researcher level, and certainly represent significant change at the institutional/infrastructural level. It is imperative that both researchers and institutions understand the nature of and accommodate these changes.

Keywords: Conflicts of Interest; PHS-Funded Research; HMORN Administrative and Technical

$$
\text { doi:10.3121/cmr.2012.1100.cb5-02 }
$$

CB5-03:

Beyond the IRB: Reviewing Projects With Respect to Organizational Risk and Benefits

Hal Luft ${ }^{1}$

${ }^{1}$ Palo Alto Medical Foundation, Sutter Health

Background/Aims: Institutional Review Boards (IRBs) are charged with protecting human subjects, balancing experimental risks against potential benefits. IRB members sometimes raise concerns that project findings may create risks for an organization. Even if the concerns are legitimate, IRBs have neither the responsibility nor the appropriate expertise to address them These concerns arise not from research processes potentially addressable by changing the study methods, but from potential findings. Ex ante reviews are speculative; ensuring low probability scenarios never occur means avoiding most delivery system research. Ex post review and approval allows projects to begin, but are generally unacceptable to researchers, funders, and journals. A two-phase process incorporating ex post reviews, but not approvals, offers a solution. Methods: At the request of its Research Institute (RI), the Palo Alto Medical Foundation (PAMF) established an Advisory Committee Concerning Organizational Risk and Dissemination (ACCORD). Members include PAMF's Chief Risk, Compliance, and Operations Officers, the Vice President for Strategic Planning and Business Development, Regional General Counsel, Director of Communications and Public Affairs, and the medical group's Chief Medical Quality and Medical Officers. Input from the 8-person ACCORD occurs at two major junctures of the project's lifebefore submission to a funding agency (or if no external funds are requested, before submission to the IRB) and if requested, before manuscript submission. Results: An Organizational Risk Assessment (ORA) form elicits from investigators potential risks in specific areas and how they can be mitigated or managed. Questions focus on organizational image, business strategy, legal/regulatory issues, compliance, quality, operations, and the medical group. ACCORD members raise concerns they have, offer suggestions, and score the project from "serious concerns-let's discuss" to "risks identified and managed" to "potential value to us." The first triggers before project commencement a discussion between those raising the concerns and the investigator. The RI director can suggest risk mitigation and management strategies. The ACCORD may ask to comment on project manuscripts before submission, or just be notified before publication. The process is being implemented; early results will be discussed. Discussion: Delivery system research entails various organizational risks. A proactive two-phase advisory process may efficiently balance organizational concerns and researcher autonomy.

Keywords: IRB Processes; Organizational Risk; HMORN Administrative and Technical

doi:10.3121/cmr.2012.1100.cb5-03

\section{Learning Health Care Systems}

PS2-12:

Rapid Cycle Clinical Information Technology Innovations to Monitor and Tailor Pediatric Care for Obesity Interventions

Margaret Rukstalis ${ }^{1}$; Sanjay Udoshi ${ }^{1}$; Joseph Leader ${ }^{1}$; Sam Kao ${ }^{1}$; William Cochran $^{1}$

\section{${ }^{1}$ Geisinger Health System}

Background/Aims: Pediatric obesity is the most common, preventable chronic disease. Multiple guidelines concur that systematic, routine strategies are required to monitor body mass index (BMI) to identify overweight ( $>85$ th $\%$ BMI) and obese (>95th\% BMI) children (ages 2-18), to intervene and provide follow up. This study reports on how health system leaders use electronic health records (EHR) and clinical decision intelligence support (CDIS) to track age specific BMI\% and tailor pediatric care for obesity interventions. Methods: Between 1996-2001, Geisinger Health System adopted a common EHR platform to link 41 primary care clinics in 31 rural and urban counties in central-NE PA. In 2007, Geisinger created CDIS as an EHR-enterprise data warehouse. Every 24 hours, CDIS IT extracts data from our EHR, including heights and weights captured in industry standard format [(Health Level 7 (HL7)]. We used the CDC SAS scripts from the CDC growth chart website to calculate $\mathrm{BMI} \%$ for age, sex for 2010 well child visits (WCV). Results: In 2010, 32,443 unique children had WCV. BMI\% was recorded for 92\%: 5,255 (16\%) were overweight defined as BMI > 85$94.99 \%$ and 5,413 (17\%) were obese (BMI $>95$ th\%). A majority of the obese children (80\%) had 2-16 visits with BMIs $>95$ th\%. Discussion: In 2010, 33\% of children ages 2-18 with documented EHR WCV had BMI $>85$ th $\%$ overweight/obese. These results led to the 2011 GHS initiative where each night, CDIS extracts data to calculate $\mathrm{BMI} \%$ and update a list of obese children that is monitored by physician leaders and practice sites. CDIS then loads an alert to fire at the child's next visit to provide physicians with 